Winter Meeting - Joint meeting between the Nutrition Society and the Royal Society of Medicine, 11-12 December 2012,

\title{
Probiotic Lactobacilli stimulate avian beta-defensin 9 expression in cultured chicken small intestinal epithelial cells
}

\author{
G. H. Li, Z. M. Hong, Y. J Jia, J. M. You, J. H. Zhang and B. S. Liu \\ College of Animal Science and Technology, Jiangxi Agricultural University, Nanchang, China
}

Beta-defensins, which are gene-encoded cationic peptides, are widely distributed in various tissues, especially in epithelium of skin, digestive, respiratory, and urogenital tracts ${ }^{(1)}$. In addition to their direct antimicrobial activities, beta-defensins play an important role in the integration of the innate and acquired immune responses ${ }^{(2)}$. The avian beta-defensin 9 (AvBD9) plays an important role in maintaining the homeostasis of gastrointestinal microflora and intestinal immune system ${ }^{(3)}$. The probiotic Lactobacilli, predominantly clonized in the chicken small intestine, interact with epithelial cells. The present study was conducted to investigate whether the probiotics can induce the expression of AvBD9 during the probiotic interaction with chieken intestinal epithelial cells (IEC).

Seven strains of probiotic Lactobacilli were selected to investigate the effects of lactobacillus stimuli on the AvBD9 mRNA expression in cultured intestinal epithelial cells and to compare the induction differences of AvBD9 expression between different probiotic bacterial strains. AvBD9 expression differences were observed among probiotic bacteria and different stimulation concentrations of bacteria. Lactobacillus fermentum F6, Lactobacillus rhamnosus MLG $_{\mathrm{A}}$, Lactobacillus rhamnosus MB12 and Lactobacillus plantarum SJ could stimulate AvBD9 mRNA expression in a different extent. From a comparison of all tested lactobacillus strains, the Lactobacillus ramous $\mathrm{MLG}_{\mathrm{A}}$ has the strongest capability to promote the AvBD9 expression. Heat-inactivated Lactobacillus ramous MLG $_{\mathrm{A}}$ also up regulated the expression of AvBD9, and showed stronger induction response than live bacteria (Fig. $1 ; P<0.05$ ). AvBD9 peptide was detected by Western blot in the supernatants of cultured epithelial cells treated with L. ramous LGA (Fig. 2), indicating that AvBD9 peptide releases into extracellular medium and exerts bactericidal action. This study reveals the new possible functional mechanism by which the probiotic Lactobacilli exert their beneficial effects to the host through the antimicrobial peptide expression in the small intestinal epithelium.

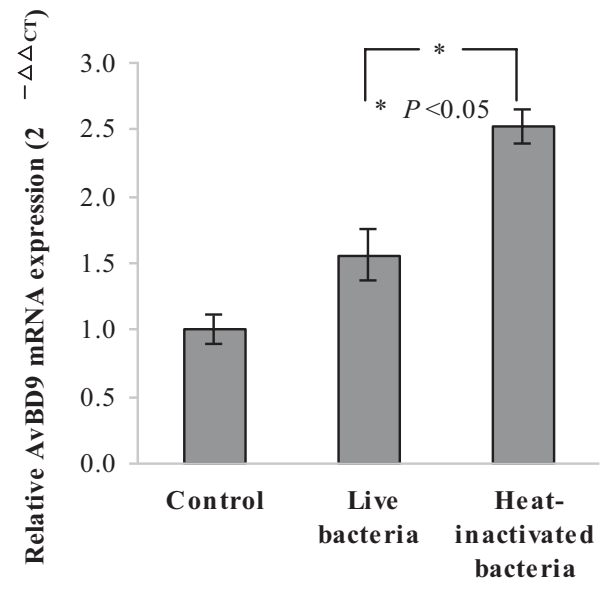

Fig. 1. AvBD9 mRNA expression in IEC.

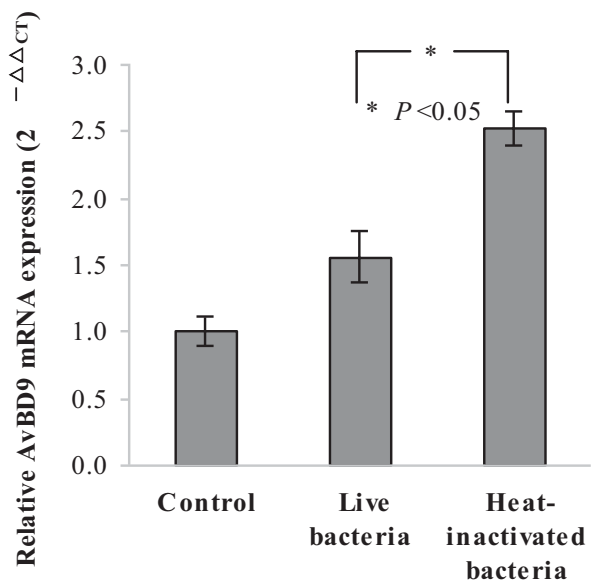

Fig. 2. AvBD9 peptide in culture supernatants detected by Western blot.

This work was supported by grants from National Natural Science Foundation of China (No. 30800791).

1. Zasloff M (2002) Nature 415, 389-395.

2. Steinstraesser L, Kraneburg U, Jacobsen F et al. (2011) Immunobiology 216, 322-333.

3. van Dijk A, Veldhuizen EJA, Haagsman HP (2008) Vet Immunol Immunopathol 124, 1-18. 